\title{
Striatal Dopamine Depletion Induces Forelimb Motor Impairments and Disrupts Forelimb Movement Representations within the Motor Cortex
}

\author{
Emily K. Plowman ${ }^{\mathrm{a}, *}$, Nagheme J. Thomas ${ }^{\mathrm{b}}$ and Jeffrey A. Kleim ${ }^{\mathrm{b}}$ \\ ${ }^{a}$ Department of Communication Sciences and Disorders, University of South Florida, FL, USA \\ ${ }^{\mathrm{b}}$ School of Biological and Systems Health Engineering, Arizona State University, AZ, USA
}

\begin{abstract}
While limb motor deficits of Parkinson's disease (PD) are well characterized, the effects of striatal dopamine depletion on the motor cortex is poorly understood. We therefore aimed to 1) examine the effects of striatal dopamine depletion on forelimb function and cortical motor map topography and 2) explore potential relationships between forelimb function and cortical movement representations in an animal model of PD. Twenty-four male Long Evans rats were randomized to control or 6-hydroxydopamine (6-OHDA) groups. Animals in the 6-OHDA group underwent four unilateral 6-OHDA infusions into the striatum to induce striatal dopamine depletion. Four weeks later, animals were tested on a comprehensive battery of behavioral limb motor tasks followed by intracortical microstimulation to derive high-resolution topographic maps of forelimb movement representations. Standard tyrosine hydroxylase $(\mathrm{TH})$ immunohistochemistry was performed and near infrared densitometry analysis utilized to assess TH depletion. Unilateral striatal dopamine depletion induced significant reductions in limb motor function that were reflected neurophysiologically as a reduction in cortical forelimb movement representations. Voluntary forelimb use, pasta handling, sunflower seed manipulation, and forelimb motor maps were all significantly impaired in 6-OHDA animals. A positive correlation was observed between forelimb function and motor map size, as well as two negative correlations between TH depletion with 1) motor map size and 2) forelimb function. The results clearly show how dysfunction within the basal ganglia thalamocortical loop resulting from nigrostriatal dopamine depletion disrupts corticospinal function.
\end{abstract}

Keywords: 6-Hydroxydopamine, 6-OHDA, parkinson's disease, intracortical microstimulation, rodent, limb motor, motor map, forelimb function

\section{INTRODUCTION}

Parkinson's Disease (PD) is a chronic, progressive and non-curable neurodegenerative disease associated with substantial morbidity, increased mortality and

*Correspondence to: Emily K. Plowman, Ph.D., CCC-SLP, Assistant Professor, Department of Communication Sciences \& Disorders, PCD 1017, University of South Florida, Tampa, FL 33620, USA. Tel.: +813 974 2397; Fax: +813 974 0822; E-mail: plowman@usf.edu. high economic burden [1]. Approximately 1.5 million Americans are currently diagnosed with PD at a cost of $\$ 23$ billion dollars annually [1]. Hallmark characteristics of PD include resting tremor, bradykinesia, rigidity, and postural instability that are the clinical offprint of dysfunction within the basal ganglia thalamocortical (BGTC) loop. The basal gangliathalamocortical circuit model of PD contends that loss of nigrostriatal dopamine results in hyperactivity of inhibitory basal ganglia output to thalamic nuclei. This 
in turn inhibits thalamocortical projections and reduces cortical excitability contributing to the hypokinetic features of PD [2]. Support for this hypothesis comes from functional imaging studies in PD patients that reveal hypoactivity in the premotor and prefrontal cortex [3-6]. Further, neuronal recording studies in animal models of parkinsonism [7,8] and in humans with dyskinesias [9] show aberrant activity within BGTC circuits. Lesions of the basal ganglia or thalamus that prevent excess inhibition within in the BGTC loop are highly effective in treating bradykinesia, rigidity and tremor in animals with experimental parkinsonism [10] and in PD patients [11] and decreases in movement related cortical activity can be partially reversed with dopamine replacement therapy [12-14]. The effects of such persistent changes in cortical excitability observed in PD on the functional organization of motor cortical areas are poorly understood. The finding that intrastriatal dopamine depletion reduces metabolic activity and immediate early gene expression within the rodent motor cortex $[15,16]$ suggests that there are enduring effects of imbalanced excitability on motor cortex function that may contribute to limb motor dysfunction. The investigation of cortical motor maps in response to striatal dopamine depletion using intracortical microstimulation (ICMS) in an animal model of PD provides a unique opportunity to critically evaluate the relationship between striatal dopamine depletion and motor cortex function.

Two investigators have utilized ICMS to examine the effects of dopamine depletion on topographical forelimb motor maps in rodents. Metz and colleagues [17] first examined the effects of unilateral lesions of 6-Hydroxydopamine (6-OHDA) into the medial forebrain bundle (MFB) and reported preserved motor map topography despite forelimb motor impairments. More recently, Brown and colleagues [18] studied the effects of haloperidol as well as unilateral and bilateral 6OHDA infusions into the striatum on forelimb motor map topography. These authors reported significant reductions in motor map area following haloperidol administration or bilateral 6-OHDA infusions and concluded that bilateral loss of dopamine is required to induce significant changes in motor map topography. However, the relationship between these changes and limb motor function is unknown. Thus, future investigation is warranted to directly examine relationships between motor behavior and motor maps in dopamine depleted systems. The purpose of the present study was to 1) examine the effects of unilateral striatal dopamine depletion on forelimb function and cortical motor map topography and 2) explore potential relationships between forelimb motor function and forelimb cortical movement representations.

\section{METHODS}

\section{Animals}

Twenty-four male Long Evans hooded rats (approximately 180 days old with body weight of 425-525 g) were used in this experiment (Harlan, Indianapolis, Ind., USA). Animals were doubly housed in Plexigas cages $(36 \mathrm{~cm}$ long, $20 \mathrm{~cm}$ wide and $21 \mathrm{~cm}$ deep) with sawdust bedding and maintained on a 12:12 h light:dark cycle with controlled temperature and humidity. All behavioral testing occurred during the light period of the cycle and the experiment was conducted in compliance with the guidelines of the University of Florida Animal Care and Use Committee. Rats were given seven days to acclimatize to the housing environment and then randomly assigned to either a 6-OHDA $(n=12)$ or control group $(n=12)$. Rats received ad libitum rat chow and water throughout the entire study.

\section{6-OHDA infusions}

Animals assigned to the 6-OHDA group received four unilateral 6-OHDA infusions into the left hemisphere following the protocol of Kirik et al. [19]. Rats were anaesthetized with isoflurane (4\% induction, $1.5 \%$ maintenance; VIP-3000 Vaporizer, Matrix, Orchard Park, NY) and placed in a stereotaxic instrument (Kopf, Tujunga, CA) with the incisor bar set to skull flat. Infusion coordinates relative to bregma were: $(\mathrm{AP}+1.3 \mathrm{~mm}, \mathrm{ML}-2.6 \mathrm{~mm}$; $-5.0 \mathrm{~mm} \mathrm{DV}) ;(\mathrm{AP}+0.9 \mathrm{~mm}, \mathrm{ML}-3.0 \mathrm{~mm} ;-5.0 \mathrm{~mm}$ DV); (AP $-0.4 \mathrm{~mm}, \mathrm{ML}-4.2 \mathrm{~mm} ;-5.0 \mathrm{~mm} \mathrm{DV})$; (AP $-1.3 \mathrm{~mm}, \mathrm{ML}-4.5 \mathrm{~mm} ;-5.0 \mathrm{~mm} \mathrm{DV}$ ) in the left hemisphere. At each infusion site, a microbur $(0.45 \mathrm{~mm}$ in diameter) was made through the skull and a micropipette was lowered into the striatum at each coordinate. Using a computer assisted nanoinject microinfusion pump (World Precision Instruments, Sarasota, FL), $7 \mu \mathrm{g}$ (free base weight) of 6-OHDA hydrochloride (Sigma, Oakville, ON) dissolved in $0.1 \%$ ascorbic acid was infused at a rate of $1 \mu \mathrm{l} / \mathrm{min}$. The micropipette was left in place for 5 minutes to aid infusate diffusion before being slowly retracted from the brain. Following the four intrastriatal infusions, $4 \mathrm{cc}$ of Ringers solution given subcutaneously (s.c.), the scalp sutured and betadine applied to the suture site. 


\section{Protocol}

Animals remained in their home cages for fourweeks following infusion before initiating the test battery. Behavioral testing included cylinder exploration, vermicelli handling and sunflower seed opening tasks and was completed by blinded examiners.

\section{Cylinder test}

Animals were placed into a transparent cylinder $(20 \mathrm{~cm} \times 30 \mathrm{~cm})$ for 5 minutes and video recorded for subsequent analysis of forelimb use during vertical exploration. The primary outcome variable for the cylinder test was the cylinder asymmetry ratio (CAR) as described by Shallert et al. [20]. The CAR was calculated as an index of voluntary forepaw use $[$ (right touches $+1 / 2$ number of both $) /($ right touches + left touches + both) $\times 100]$.

\section{Sunflower seed opening}

To test object manipulation abilities, animals were placed into a clear plastic arena with five sunflower seeds located in the upper right hand corner. A mirror was angled at a 120 degree angle at the back of the enclosure to allow visualization of sunflower manipulation in the instance that an animal faced away from the experimenter and towards the back of the enclosure. To acclimate animals to testing materials, sunflower seeds were included in their diet several days leading up to testing. The primary outcome measure for sunflower seed testing was total manipulation time (TMT) and defined as the total time spent manipulating, opening and placing the seed into the mouth (starting the moment the animal touched a seed and ending the second the animal dropped the shell and released the seed into the mouth) and represented the cumulative and total time across all five trials. The secondary outcome measure for sunflower seed testing was the total number of shell pieces (TSP) produced to open the five seeds. Animals were tested over two consecutive days and a two-day average TMT and TSP calculated.

\section{Vermicelli handling test}

Animals were presented with five $7 \mathrm{~cm}$ uncooked vermicelli strands $(1.5 \mathrm{~mm}$ diameter; $0.15 \mathrm{~g} /$ piece; Mueller brand, distributed by American Pasta Co., Kansas City, MO) in their home cage and video recorded for subsequent analyses. To acclimate animals to pasta handling, they were given five strands of vermicelli in their home cages for several days prior to testing. The primary outcome measure for the vermi- celli handling test was the vermicelli asymmetry ratio (VAR) as described by Allred et al. [21]. The VAR $(\%)$ is defined as the [(number right adjustments/total number of adjustments) $x$ 100]. Time to eat (beginning when the pasta piece was grasped and ending when piece was released by the paws and disappeared into the mouth in seconds) was a secondary outcome measure for this task. For data analysis, the mean across the five trials was used.

\section{Intracortical microstimulation}

Within two weeks of the final training session, Intracortical Microstimulation (ICMS) was used to generate detailed maps of forelimb regions [22, 23] within the motor cortex ipsilateral to the 6-OHDA infusions. Prior to surgery animals were anesthetized with ketamine hydrochloride $(70 \mathrm{mg} / \mathrm{kg}$ i.p.) and xylazine $(5 \mathrm{mg} / \mathrm{kg}$ i.p.) with supplemental doses administered as needed. Careful attention was paid to breathing rate and whisking in order to determine when animals required supplemental doses of anesthetic. Further, stimulation of the internal capsule (see below) was also used to dissociate stimulation sites that were non-responsive due to anesthetic level versus experimental condition. Under sterile conditions, a craniotomy was performed over the left (ipsi-lesional) motor cortex and a small puncture made to the cisterna magna to prevent edema prior to removing the skull and dura. The exposed cortex was then covered in warm saline $\left(37^{\circ} \mathrm{C}\right)$ and a digital image of the cortical surface taken and saved in CANVAS 10.0 (ACD Systems, Vancouver, BC) on a Macintosh Computer (Cupertino, CA). A glass microelectrode (controlled by a hydraulic microdrive) was used to make systematic penetrations across the cortex using the cortical surface image with a $300 \mu \mathrm{m}$ superimposed grid as a guide. At each penetration site, the electrode was lowered to approximately $1550 \mu \mathrm{m}$ (corresponding to cortical layer V). Stimulation consisted of thirteen, $200 \mu$ s cathodal pulses delivered at $350 \mathrm{~Hz}$ from an electrically isolated stimulation circuit at a rate of $1 \mathrm{~Hz}$. Animals were in a prone position with the contralateral limb supported. Sites where no movement was detected at $\leq 60 \mu \mathrm{A}$ were coded unresponsive. A secondary stimulation procedure within the ipsilateral internal capsule in 6-OHDA animals was performed in order to ensure nonresponsive sites, were not due to altered anesthesia levels. The microelectrode was placed into the internal capsule (3.0 AP, 3.0 ML, $7.0 \mathrm{DV}$ ) and stimulation passed at $40 \mu \mathrm{A}$. Movement of the forelimb was then confirmed. The primary outcome variable was total motor map area $\left(\mathrm{mm}^{2}\right)$ for proxi- 
mal and distal forelimb sites (TMA). The secondary outcome variable was the mean stimulation threshold (MST, $\mu \mathrm{A})$.

\section{Immunohistochemistry}

Immediately following mapping, animals were perfused intracardially with $200 \mathrm{ml}$ of phosphate buffered saline solution followed by $400 \mathrm{ml}$ of $4 \%$ paraformaldehyde and decapitated for brain extraction. Brains were post fixed in $4 \%$ paraformaldehyde for 24 hours and then cut into $40-\mu \mathrm{m}$-thick coronal sections using a freezing microtome. Floating sections were washed with $0.01 \mathrm{M}$ PBS and then treated for 15 min with $0.5 \% \mathrm{H}_{2} \mathrm{O}_{2}+10 \%$ methanol in $0.01 \mathrm{M}$ PBS. Sections were preincubated with $3 \%$ normal horse serum (NHS)+0.1\% Triton X-100 in $0.01 \mathrm{M}$ $\mathrm{PBS}$, and then incubated overnight at room temperature with a $1: 2000$ dilution of a mouse anti-TH antibody (Chemicon, Temecula, CA). Following incubation, tissue was washed and incubated for 2 hours at room temperature with a secondary antibody directed against the species in which the primary antibody was raised. The reactions were then incubated with a near infrared secondary antibody for TH densitometry, IRdye 800 (LI-COR Biosciences, Lincoln, NE). Sections were mounted on subbed slides, dehydrated in ascending alcohol concentrations, cleared in xylene, and coverslipped in permount in a dark room.

\section{Densitometry}

Serial TH-labeled sections were scanned at a resolution of $21 \mu \mathrm{m}$ and a 5.5 sensitivity setting on a LI-COR Odyssey scanner (LI-COR Biosciences). Using the Odyssey Infrared imaging system Application Software (Version 3.0), striatal sections were outlined and the value of the integrated intensity obtained from the right and left striatum of each section derived. $\mathrm{TH}$ depletion was calculated for each animal using the following formula: [ $\varepsilon$ left integrated intensity/ $\varepsilon$ right integrated intensity) $\times 100]$ following the protocol of Manfredsson et al.[24].

\section{Statistical analyses}

Behavioral and mapping data were analyzed using a series of independent student $t$ tests between control and 6-OHDA groups. Results were confirmed with the non-parametric Mann-Whitney U test. For these behavioral analyses, only those animals with TH depletion greater then $50 \%$ were included in the 6-OHDA group (see next section). Pearson's Correlation analyses were also performed between $\mathrm{TH}$ depletion levels (\%), forelimb behavioral outcomes and forelimb motor map area. For those correlation analyses involving TH depletion, only those animals injected with 6-OHDA were included in the analysis due to no range in depletion level in control animals. For all statistical tests, $\alpha$ was set at 0.05 and SPSS 18.0. (Chicago, IL) was used to compute all data. All descriptive statistics are reported as means \pm S.E.M unless otherwise indicated.

\section{RESULTS}

\section{Densitometry}

Three animals were excluded from the 6-OHDA group due to insufficient $\mathrm{TH}$ depletion $(<50 \%)$. Of those animals included in the 6-OHDA group $(n=9)$, densitometry analyses revealed that mean TH depletion was $81 \%$ (range $=51-100 ; \mathrm{SD}=18.74$; $\mathrm{SEM}=6.62$ ). Fluorescent immunoreactivity staining revealed that striatal 6-OHDA infusion resulted in loss of TH-positive cells in the striatum. Fig. 1B illustrates a representative lesion producing loss of dopaminergic innervation in the ipsi-lesional striatum.

\section{Behavioral impairments}

The 6-OHDA group displayed significant impairments across all behavioral tasks (Table 1). For cylinder testing, voluntary use in the right forelimb (CAR) decreased from $50.86 \%( \pm 4.11)$ in the control group to $16.23 \%( \pm 5.19)$ in the 6-OHDA group $(p<0.001)$. During sunflower seed testing, TMT increased from $63.60 \mathrm{sec}$ (9.23) in the control group to $117.20 \mathrm{sec}$ $( \pm 24.31)$ in the 6-OHDA group and TSP increased from $12.40(0.62)$ in the control group to $18.00(1.38)$ in the 6-OHDA groups $(p<0.05)$. During vermicelli pasta handling, use of the right forelimb (VAR) decreased from $49.45 \%( \pm 1.62)$ in the control group to $39.2 \%$ $( \pm 3.23)$ in the 6-OHDA group $(p<0.01)$. No differences were observed for the average time to eat the five vermicelli strands in comparison to controls $(p>0.05)$.

\section{Motor map topography}

No significant differences in the amounts of ketamine or xylazine as a function of body weight and duration of surgery were noted between control and 6OHDA groups. Amounts of ketamine were $1.57 \mathrm{mg} /$ $\mathrm{kg} / \mathrm{min}( \pm 0.12)$ for controls and $1.53 \mathrm{mg} / \mathrm{kg} / \mathrm{min}$ $( \pm 0.14)$ for the 6-OHDA group $(p>0.05)$. Amounts 

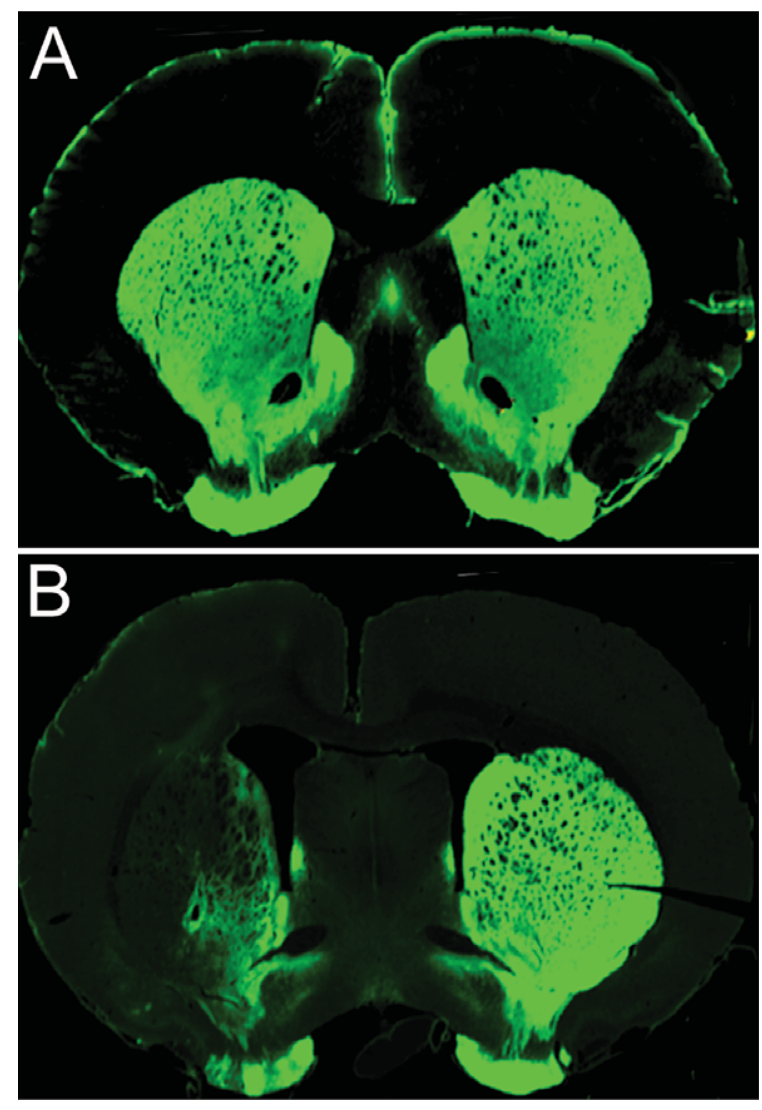

Fig. 1. Representative TH stained 40- $\mu \mathrm{m}$ coronal sections through the striatum of a control (A) and unilateral depleted 6-OHDA animal. (B). 6-OHDA animals show a significant decrease in TH staining within the striatum.

of xylazine were $0.89 \mathrm{mg} / \mathrm{kg} / \mathrm{min}( \pm 0.0075)$ for controls and $0.03 \mathrm{mg} / \mathrm{kg} / \mathrm{min}( \pm 0.0073)$ for the 6-OHDA group $(p>0.05)$.

Representative motor maps for a control and 6-OHDA animal are shown in Fig. 1A and 1B respectively. A significant reduction in total forelimb motor map area was demonstrated for the 6-OHDA group $(p<0.05)$ (see Fig. 2C). No significant differences were noted in the size of proximal or distal forelimb maps $(p>0.05)$ or in forelimb area movement distribution ( $p>0.05$, Fig. 2D). Mean threshold current intensities required to elicit forelimb movements were $29.25 \mu \mathrm{A}( \pm 1.51)$ for controls and $26.54 \mu \mathrm{A}( \pm 4.77)$ for the 6-OHDA group $(p>0.05)$.

\section{Relationship between forelimb function and forelimb motor maps}

A significant positive correlation was revealed between forelimb motor map area and cylinder forelimb asymmetry ratio $(r=0.48 p<0.05)$. In general, the greater the voluntary forelimb use, the larger the forelimb motor map observed (Fig. 3A). No other relations were revealed between map area and behavioral testing measures $(p>0.05)$.

\section{Relationship between TH depletion forelimb function and forelimb motor maps}

A significant negative correlation was observed between $\mathrm{TH}$ depletion and cylinder forelimb asymmetry $(r=-0.85, p<0.001)$ as well as between $\mathrm{TH}$ depletion and forelimb motor map area $(r=-0.45$, $p<0.05$ ) (Figs. 3B and $3 \mathrm{C}$ respectively). The greater the dopamine depletion, the lower voluntary forelimb use in the contralateral limb and the smaller the forelimb motor map area observed.

\section{DISCUSSION}

This study demonstrates a clear relationship between striatal dopamine depletion, impairments in limb motor function and concomitant reductions in forelimb movement representations in the motor cortex. Forelimb use during voluntary exploration, pasta handling and sunflower seed manipulation, were all

Table 1

Summary of behavioral testing results

\begin{tabular}{|c|c|c|c|c|c|c|}
\hline \multirow[t]{2}{*}{ Motor Test } & \multirow[t]{2}{*}{ Measure } & \multicolumn{2}{|c|}{ Control } & \multicolumn{2}{|c|}{ 6-OHDA } & \multirow[b]{2}{*}{$p$ Value } \\
\hline & & Mean & (S.E.M) & Mean & (S.E.M) & \\
\hline Cylinder & CAR $(\%)$ & 50.86 & $(4.11)$ & 16.23 & $(5.19)$ & $0.001 *$ \\
\hline \multirow[t]{2}{*}{ Sunflower Opening } & TMT (sec) & 63.60 & $(9.23)$ & 117.20 & $(24.31)$ & $0.02 *$ \\
\hline & TSP & 12.40 & $(0.62)$ & 18.00 & $(1.38)$ & $0.001 *$ \\
\hline \multirow[t]{2}{*}{ Vermicelli Handling } & $\operatorname{VAR}(\%)$ & 49.45 & (1.62) & 39.12 & $(3.23)$ & $0.007 *$ \\
\hline & Time to eat (sec) & 75.90 & (11.72) & 85.16 & (18.54) & 0.66 \\
\hline
\end{tabular}

$\mathrm{CAR}=$ cylinder asymmetry ratio. $\mathrm{VAR}=$ vermicelli asymmetry ratio. $\mathrm{TMT}=$ total manipulation time. $\mathrm{TSP}=$ total shell pieces. * significant between groups difference $(p<0.05)$. 
A
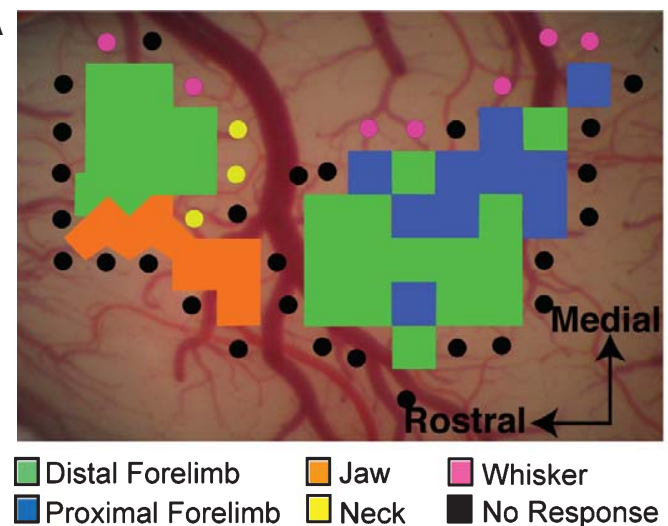

B

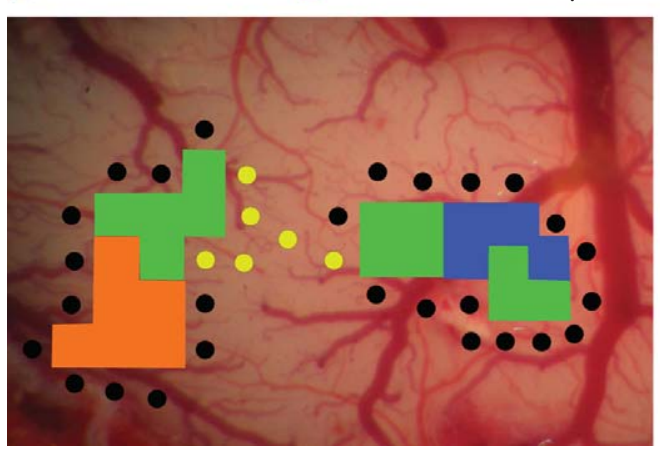

C Motor Map Area
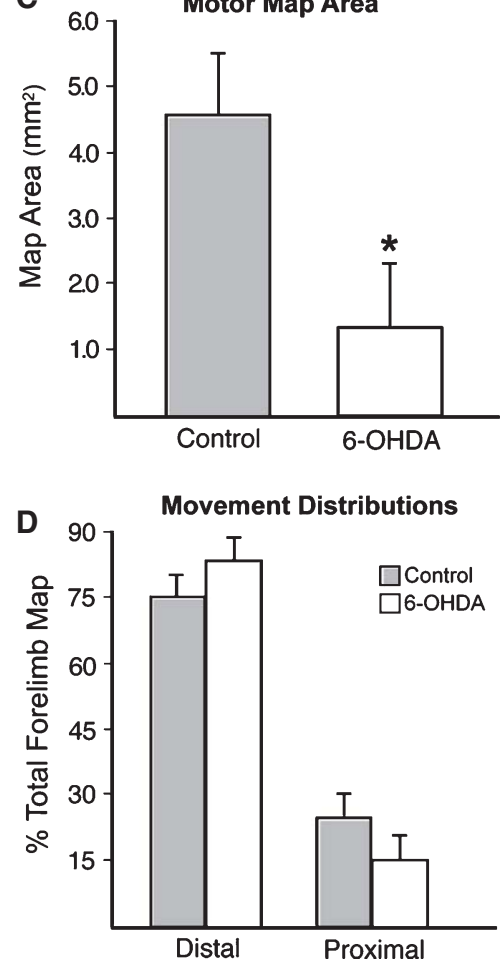

Fig. 2. A. Representative motor map for a control animal. B. Representative motor map for a unilateral 6-OHDA animal. C. A significant reduction in forelimb motor map area was observed for the 6-OHDA group $(p<0.05)$. D. The relative proportion of distal versus proximal movement distributions did not differ between groups $(p<0.05)$.

seen to be significantly impaired following unilateral striatal dopamine depletion. This suggests a generalized impairment in limb motor function and is consistent with the upper extremity impairments observed in PD patients. A reduction in the amount of forepaw use was observed in both the cylinder and vermicelli handling tasks as reflected by significantly lower asymmetry ratios than the control group and a greater amount of time being required to open sunflower seeds. Further, a significant relationship was found between the amount of voluntary forelimb use in the affected limb (cylinder asymmetry ratio) and forelimb cortical movement representations as well as TH depletion suggesting that the level of functional impairment is related to integrity of the forelimb motor maps and degree of dopamine depletion.

Similar to forelimb function, forelimb cortical motor maps were also impaired following unilateral striatal dopamine depletion. Specifically, forelimb motor map area was significantly smaller in the 6-OHDA animals compared to controls. No differences were noted, however, in forelimb motor thresholds or specific movement distributions. There are several possible explanations for the observed loss of movement representations. First, motor map topography is highly sensitive to changes in synapse number and strength within the motor cortex. Manipulations that reduce synapse number [25] or decrease synaptic strength [26] also reduce motor map size. The observed loss of movement representations in 6-OHDA animals may therefore result from a loss of synapse number and/or strength within the motor cortex. In keeping with the BGTC model, increased inhibition of the thalamus from striatal output nuclei could result in a weakening of thalamocortical afferents. Because ICMS induces movement via transynaptic activation of corticospinal neurons [27], a degradation of thalamocortical synapse could contribute to a reduction in motor map area. In support of this hypothesis, forelimb movements could be readily elicited in 6-OHDA animals via stimulation of the internal capsule. This stimulation protocol elicits movement by directly stimulating corticospinal axons and is not dependent upon transynaptic activation of cortical pyramidal neurons. 
A
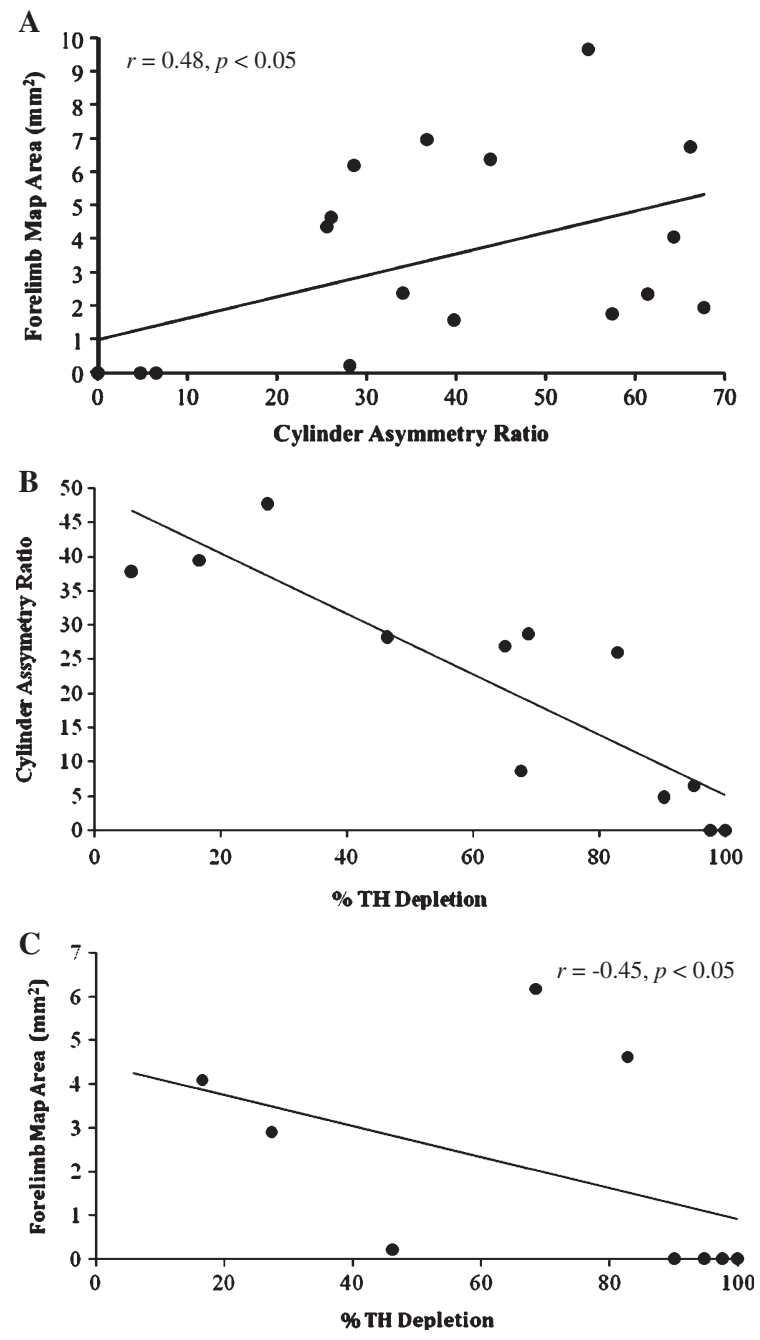

Fig. 3. A. Scatterplot depicting the relationship between total forelimb motor map area and cylinder asymmetry ratio. Animals in both the control and 6-OHDA group were used in this analysis. Note that complete motor maps were not obtained for three animals resulting in three missing data points. B. Scatterplot depicting the relationship between cylinder asymmetry ratio and percentage Tyrosine Hyroxylase depletion. Only those animals injected with 6-OHDA $(n=12)$ were included in this analysis. C. Scatterplot depicting the relationship between total forelimb motor map area and percentage Tyrosine Hyroxylase depletion. Only those animals injected with 6-OHDA $(n=12)$ were included in this analysis. Note that complete motor maps were not obtained for three animals resulting in three missing data points.

This is the third report documenting effects of dopamine depletion on forelimb topographic movement representations. Contrary to our results, Metz and colleagues [17] reported preserved forelimb motor map size in naïve and skilled reach trained rats following unilateral lesions to the MFB. A possible explanation for the observed discrepancy might be attributed to the different surgical techniques utilized for inducing striatal dopamine depletion (MFB vs. intrastriatal). While MFB lesions involve a single infusion that induces dopamine depletion within an acute time period (within 48 hours), dopamine depletion via intrastriatal lesions occurs in a gradual progression across four infusion sites and over two phases (typically complete by four weeks [19]. Thus, the mechanisms for inducing dopamine depletion within the striatum may differ between these two models (immediate vs. gradual) and may differentially affect motor cortex physiology.

Brown et al. [18] investigated effects of striatal dopamine depletion and systemic injections of the dopamine blocker haloperidol. These investigators observed a significant reduction in forelimb motor map area as well as a topographic reorganization (an increase in the size of distal to proximal forelimb movement representation) in bilaterally depleted animals and following haloperidol administration compared to control animals. Contrary to our findings, however, unilaterally depleted animals did not demonstrate significant reductions in forelimb motor map area. The $\mathrm{TH}$ depletion reported in this study, however, was mild (mean depletion level $=64.5 \%$ ) as compared to the moderate-severe depletion observed in the present study ( mean depletion level $=81 \%$ ). Our observed relationship between forelimb motor map area and $\mathrm{TH}$ depletion level therefore suggests there is a relationship between forelimb motor map area and dopamine depletion, however this relationship is not linear. It appears that the loss of motor maps requires a critical level of dopamine depletion. Fig. $3 \mathrm{C}$ shows that only after $90 \%$ dopamine depletion does motor map area become significantly reduced. This may provide an explanation for the observed differences across studies following unilateral striatal dopamine depletion.

In summary, our results demonstrate that moderatesevere unilateral striatal dopamine depletion induces significant limb motor deficits that are reflected neurophysiologically as a reduction in forelimb motor cortex movement representations. Both cylinder forelimb asymmetry ratios and forelimb motor map area were related to the level of dopamine depletion, suggesting that both forelimb function and cortical movement representations are highly dependent on striatal dopamine levels. The results provide a neural mechanism by which maladaptive changes in activity within BGTC circuits induces long term changes in the functional organization of motor cortex and limb motor impairments in PD. 


\section{ACKNOWLEDGMENTS}

This study was supported by a National Institute of Deafness and other Communication Disorders (NIDCD) training grant 1F32DC010569-01A1.

\section{REFERENCES}

[1] Weintraub D, Comella CL, \& Horn S (2008) Parkinson's disease-Part 1: Pathophysiology, symptoms, burden, diagnosis, and assessment. Am J Manag Care, 14(2), S40-S48.

[2] DeLong M \& Wichmann T (2009) Update on models of basal ganglia function and dysfunction. Parkinsonism Relat Disord, 15(3), S237-S240.

[3] Catalan MJ, Ishii K, Honda M, Samii A, \& Hallett M (1999) A PET study of sequential finger movements of varying length in patients with Parkinson's disease. Brain, 122(3), 483-495.

[4] Eidelberg D, Moeller JR, Dhawan V, Sidtis JJ, Ginos JZ, \& Strother SC et al. (1990) The metabolic anatomy of Parkinson's disease: complementary [18F] fluorodeoxyglucose and [18F] fluorodopa positron emission tomographic studies. Mov Disord, 5(3), 203-213.

[5] Rascol O, Brooks DJ, Brunt ER, Korczyn AD, Poewe WH, \& Stocchi F (1998) Ropinirole in the treatment of early Parkinson's disease: a 6-month interim report of a 5-year levodopa-controlled study. 056 Study Group. Mov Disord, 13(1), 39-45.

[6] Haslinger B, Erhard P, Kampfe N, Boecker H, Rummeny E, \& Schwaiger $M$ et al. (2001) Event-related functional magnetic resonance imaging in Parkinson's disease before and after levodopa. Brain, 124(3), 558-570.

[7] Bergman H, Wichmann T, Karmon B, \& DeLong MR (1994) The primate subthalamic nucleus. II. Neuronal activity in the MPTP model of parkinsonism. J Neurophysiol, 72(2), 507520.

[8] Escola L, Michelet T, Macia F, Guehl D, Bioulac B, \& Burbaud P (2003) Disruption of information processing in the supplementary motor area of the MPTP-treated monkey: a clue to the pathophysiology of akinesia? Brain, 126(1), 95114.

[9] Papa SM, Desimone R, Fiorani M, \& Oldfield EH (1999) Internal globus pallidus discharge is nearly suppressed during levodopa-induced dyskinesias. Ann Neurol, 46(5), 732-738.

[10] Aziz TZ, Peggs D, Sambrook MA, \& Crossman AR (1991) Lesion of the subthalamic nucleus for the alleviation of 1-methyl-4-phenyl-1,2,3,6-tetrahydropyridine (MPTP)induced parkinsonism in the primate. Mov Disord, 6(4), 288-292.

[11] Alvarez L, Macias R, Pavon N, Lopez G, Rodriguez-Oroz MC, \& Rodriguez R et al. (2009) Therapeutic efficacy of unilateral subthalamotomy in Parkinson's disease: results in 89 patients followed for up to 36 months. J Neurol Neurosurg Psychiatry, 80(9), 979-985.

[12] Rascol O, Sabatini U, Chollet F, Fabre N, Senard JM, \& Montastruc JL et al. (1994) Normal activation of the supplementary motor area in patients with Parkinson's disease undergoing long-term treatment with levodopa. J Neurol Neurosurg Psychiatry, 57(5), 567-571.
[13] Rascol O, Ferreira JJ, Thalamas C, Galitsky M, \& Montastruc JL (2001) Dopamine agonists. Their role in the management of Parkinson's disease. Adv Neurol, 86, 301-309.

[14] Buhmann C, Bussopulos A, \& Oechsner M (2003) Dopaminergic response in Parkinsonian phenotype of Machado-Joseph disease. Mov Disord, 18(2), 219-221.

[15] Rolland AS, Herrero MT, Garcia-Martinez V, Ruberg M, Hirsch EC, \& Francois C (2007) Metabolic activity of cerebellar and basal ganglia-thalamic neurons is reduced in parkinsonism. Brain, 130(1), 265-275.

[16] Steiner H \& Kitai ST (2000) Regulation of rat cortex function by D1 dopamine receptors in the striatum. J Neurosci, 20(14), 5449-5460.

[17] Metz GA, Piecharka DM, Kleim JA, \& Whishaw IQ (2004) Preserved ipsilateral-to-lesion motor map organization in the unilateral 6-OHDA-treated rat model of Parkinson's disease. Brain Res, 1026(1), 126-135.

[18] Brown AR, Hu B, Antle MC, \& Teskey GC (2009) Neocortical movement representations are reduced and reorganized following bilateral intrastriatal 6-hydroxydopamine infusion and dopamine type-2 receptor antagonism. Exp Neurol, 220(1), 162-170.

[19] Kirik D, Rosenblad C, \& Bjorklund A (1998) Characterization of behavioral and neurodegenerative changes following partial lesions of the nigrostriatal dopamine system induced by intrastriatal 6-hydroxydopamine in the rat. Exp Neurol, 152(2), 259-277.

[20] Schallert T, Fleming SM, Leasure JL, Tillerson JL, \& Bland ST (2000) CNS plasticity and assessment of forelimb sensorimotor outcome in unilateral rat models of stroke, cortical ablation, parkinsonism and spinal cord injury. Neuropharmacology, 39(5), 777-787.

[21] Allred RP, Adkins DL, Woodlee MT, Husbands LC, Maldonado MA, \& Kane JR et al (2008) The vermicelli handling test: a simple quantitative measure of dexterous forepaw function in rats. J Neurosci Methods, $\mathbf{1 7 0}$ (2), 229-244.

[22] Kleim JA, Barbay S, \& Nudo RJ (1998) Functional reorganization of the rat motor cortex following motor skill learning. J Neurophysiol, 80(6), 3321-3325.

[23] Remple MS, Bruneau RM, VandenBerg PM, Goertzen C, \& Kleim JA (2001) Sensitivity of cortical movement representations to motor experience: evidence that skill learning but not strength training induces cortical reorganization. Behav Brain Res, 123(2), 133-141.

[24] Manfredsson FP, Tumer N, Erdos B, Landa T, Broxson CS, \& Sullivan LF et al. (2009) Nigrostriatal rAAV-mediated GDNF overexpression induces robust weight loss in a rat model of age-related obesity. Mol Ther, 17(6), 980-991.

[25] Kleim JA, Bruneau R, VandenBerg P, MacDonald E, Mulrooney R, \& Pocock D (2003) Motor cortex stimulation enhances motor recovery and reduces peri-infarct dysfunction following ischemic insult. Neurol Res, 25(8), 789-793.

[26] Teskey GC, Young NA, van Rooyen F, Larson SE, Flynn C, \& Monfils MH et al. (2007) Induction of neocortical long-term depression results in smaller movement representations, fewer excitatory perforated synapses, and more inhibitory synapses. Cereb Cortex, 17(2), 434-442.

[27] Monfils MH, Plautz EJ, \& Kleim JA (2005) In search of the motor engram: motor map plasticity as a mechanism for encoding motor experience. Neuroscientist, 11(5), 471-483. 